\title{
FIRST OBSERVATIONS NEAR JUPITER BY THE JUNO WAVES INVESTIGATION
}

\author{
W. S. Kurth*, M. Imai*, G. B. Hospodarsky*, D. A. Gurnett*, \\ S. S. Tetrick*, S.-Y. Ye*, S. J. Bolton ${ }^{\dagger}$, J. E. P. Connerney \\ and S. M. Levin ${ }^{\S}$
}

\begin{abstract}
The Juno spacecraft successfully entered Jupiter orbit on 5 July 2016. One of Juno's primary objectives is to explore Jupiter's polar magnetosphere for the first time. An obvious major aspect of this exploration includes remote and in-situ observations of Jupiter's auroras and the processes responsible for them. To this end, Juno carries a suite of particle, field, and remote sensing instruments. One of these instruments is a radio and plasma wave instrument called Waves, designed to detect one electric field component of waves in the frequency range of $50 \mathrm{~Hz}$ to $41 \mathrm{MHz}$ and one magnetic field component of waves in the range of $50 \mathrm{~Hz}$ to $20 \mathrm{kHz}$. Juno's first perijove pass with science observations occurred on 27 August 2016. This paper presents some of the first observations of the Juno Waves instrument made during that first perijove. Among radio emissions, kilometric, hectometric, and decametric emissions were observed. From a vantage point at high latitudes, many of Jupiter's auroral radio emissions appear as $\mathrm{V}$-shaped emissions in frequency-time space with vertices near the electron cyclotron frequency where the emissions intensify. In fact, we present observations suggesting Juno flew through or close to as many as five or six sources of auroral radio emissions during its first perijove. Waves made in-situ observations of plasma waves on auroral field lines such as whistler-mode hiss, a common feature of terrestrial auroral regions. We also discuss observations of dust at the equator and lightning whistlers observed over mid-latitudes.
\end{abstract}

\section{Introduction}

On 27 August 2016 Juno made its first close flyby of Jupiter, Perijove 1, with all of its instruments powered on for science observations. One of those instruments, a radio and

\footnotetext{
* Department of Physics and Astronomy, The University of Iowa, Iowa City, Iowa, USA

$\dagger$ Southwest Research Institute, San Antonio, Texas, USA

$\ddagger$ Goddard Space Flight Center, Greenbelt, Maryland, USA

$\S$ Jet Propulsion Laboratory, Pasadena, California, USA
} 
plasma wave instrument called Waves, made the first survey of Jupiter's radio emissions and plasma wave phenomena at high latitudes and close range. Waves is part of a suite of instrumentation designed to explore Jupiter's polar magnetosphere, a primary objective of the Juno mission. Juno's highly eccentric orbit has an inclination of $90^{\circ}$ with its apojove near the dawn meridian at a distance of about 113 Jovian radii $\left(R_{J}=71,492 \mathrm{~km}\right)$ and perijove near $1.06 \mathrm{R}_{\mathrm{J}}$.

Before discussing data obtained near Jupiter, we mention that on approach to Jupiter Waves observed upstream plasma waves such as electron plasma oscillations and ion acoustic waves in Jupiter's foreshock beginning nearly 1 AU from Jupiter. The instrument recorded a very clear signature of the one bow shock crossing encountered by Juno on its approach [Connerney et al., 2017]. Waves also clearly identified a number of entries into Jupiter's outer magnetospheric cavity through the detection of trapped continuum radiation. A summary of bow shock and magnetopause crossings from the approach and early orbits is given by Hospodarsky et al. [2017].

For the first orbit discussed herein, the line of apsides is nearly in the jovigraphic equator. Figure 1 shows the trajectory of Juno in a dawn magnetic meridian plane and sample magnetic field lines based on a model interior magnetic field plus current sheet [Connerney et al., 1981; 1998]. Figure 2 shows the footprint of Juno as a function of time projected along the VIP4 model field onto the north and south pole in panels (a) and (b), respectively, with the southern pole viewed from the north, through the planet.

In this paper we concentrate on observations taken within about $10 R_{J}$ of Jupiter to highlight the new aspects of the perspective of the Juno Waves observations. Clearly, radio and plasma wave observations have been made by a number of spacecraft, previously including Voyager [Warwick et al., 1979; Scarf et al., 1979], Ulysses [Stone et al., 1992], Galileo [Gurnett et al., 1996], and Cassini [Gurnett et al., 2002; Hess et al., 2014] at more equatorial and more distant locations.

The Juno Waves instrument utilizes two sensors. It has an electric dipole antenna with a geometric effective length of $2.41 \mathrm{~m}$ perpendicular to the spacecraft spin axis and a single-axis search coil magnetometer that is body-mounted with its axis parallel to the spin axis. Waves analyzes the electric field spectrum from $50 \mathrm{~Hz}$ to $41 \mathrm{MHz}$ and the magnetic spectrum from $50 \mathrm{~Hz}$ to $20 \mathrm{kHz}$ using a Low Frequency Receiver and a pair of redundant High Frequency Receivers. Survey data shown herein are acquired at the rate of one electric and one magnetic spectrum every $2 \mathrm{~s}$ until just beyond perijove below $150 \mathrm{kHz}$ and every 1 second thereafter. High frequency (>150 kHz) electric spectra are obtained once per second throughout the observations presented herein. Burst mode data in the form of captured waveforms are acquired at points of interest either through commanded intervals for recording or by an on-board algorithm that assigns a quality factor based on the integrated electric field spectral density below $150 \mathrm{kHz}$. A description of the Waves instrument is provided in Kurth et al. [2017a]. 


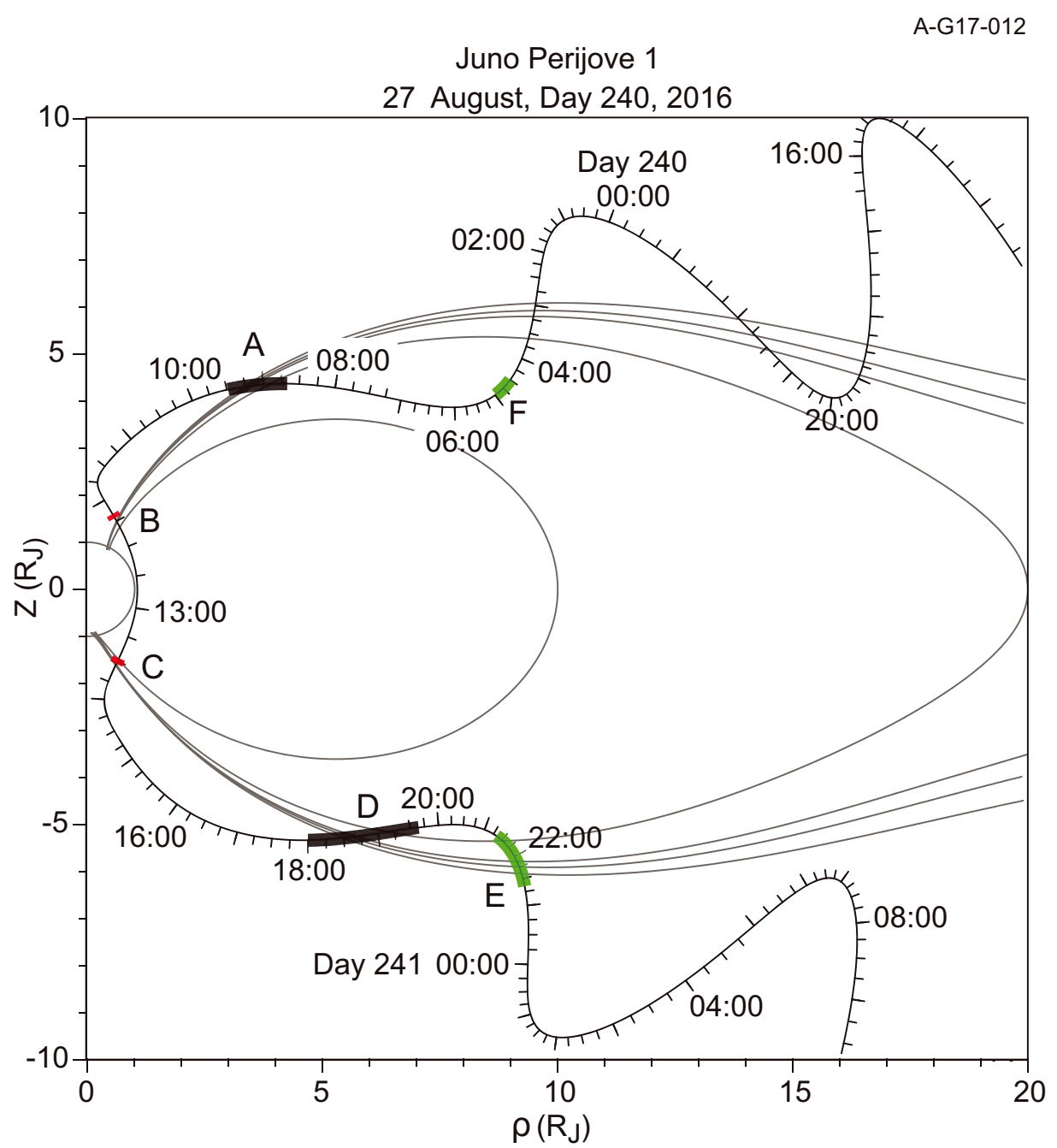

Figure 1: Trajectory of Juno in a magnetic meridian plane at $290^{\circ}$ System III longitude. The sample magnetic field lines are based on the VIP4 magnetic field model [Connerney et al., 1998] with a current sheet model from Connerney et al. [1981] having equatorial crossing distances of 10, 20,30, 40, and $50 R_{J}$. The letters identify intervals during the pass where Juno crossed through or close to auroral radio emission sources [Kurth et al., 2017b].

\section{Auroral radio emissions}

Figure 3 shows observations from Waves above $1 \mathrm{kHz}$ for a 24-hour period including Perijove 1. Auroral radio emissions are seen above the line labeled $f_{c e}=28|B|$ where the frequency is in $\mathrm{Hz}$ and the magnetic field is in $\mathrm{nT}$. The band from about $140 \mathrm{kHz}$ to $3 \mathrm{MHz}$ suffers from low sensitivity and the narrowband lines near $200 \mathrm{kHz}, 2 \mathrm{MHz}$ and $20 \mathrm{MHz}$ are interference. A series of radio emissions are found in this frequency-time spectrogram that appear as V-shaped structures. Some of them intensify as they come close to the $f_{c e}$ contour. Additionally, the electron distribution functions show upgoing loss cones and downgoing beams at times where intense radio emissions occurred near $f_{c e}$. Kurth et al. [2017b] suggested that these were either in situ observations of the sources of 


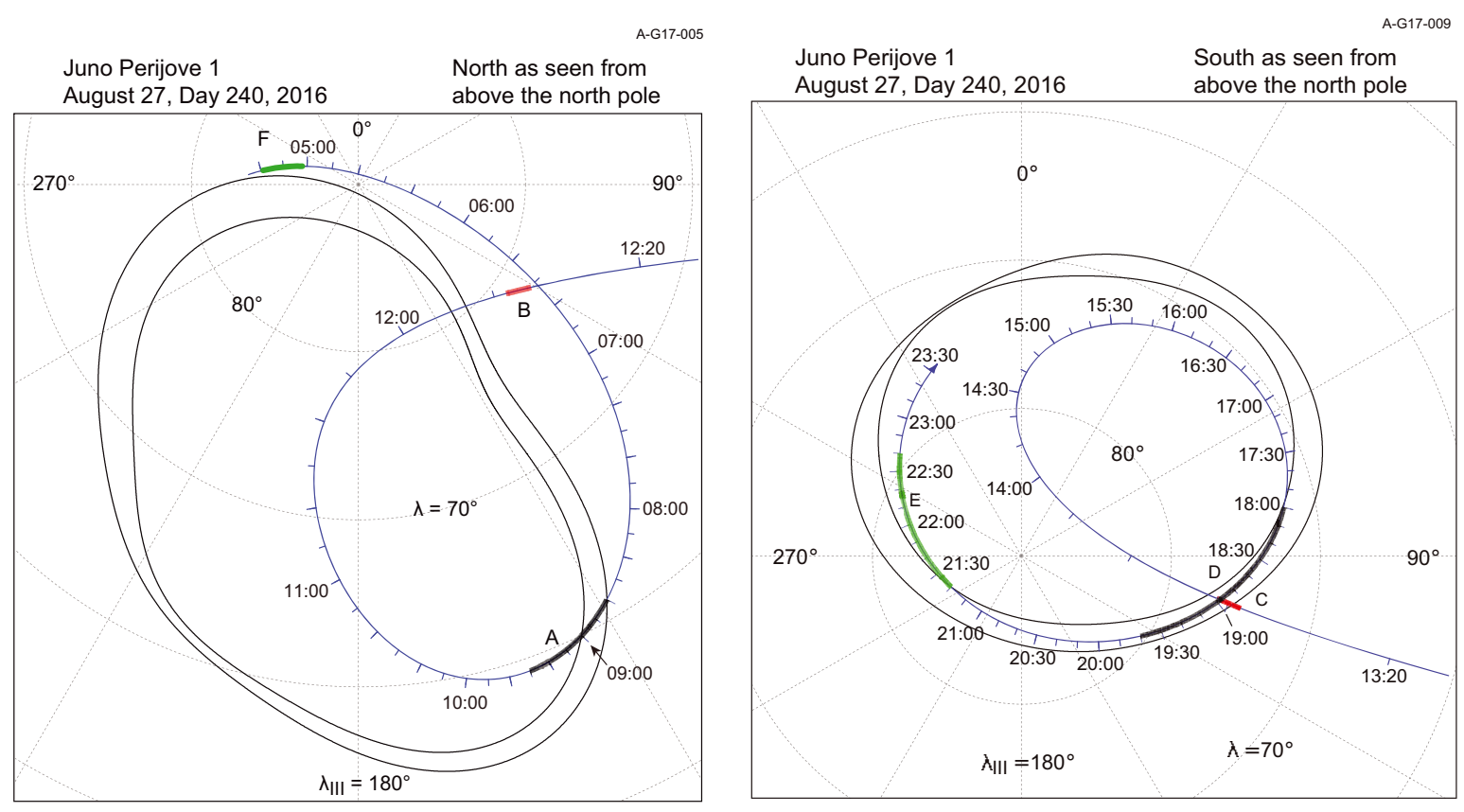

Figure 2: The footprint of Juno mapped along VIP4 [Connerney et al., 1998] field lines as a function of time. (a) The northern portion of Perijove 1. (b) The southern portion, as viewed from the north, through the planet. The double solid lines in each panel represent the statistical location of Jupiter's main auroral oval from Hubble Space Telescope observations. The letters $A-F$ are locations of possible radio sources or times when Juno was close to a source.

Jovian auroral radio emissions or close approaches to such sources. The possible source crossings are identified by letters $\mathrm{A}-\mathrm{F}$ where the identifications of $\mathrm{A}-\mathrm{E}$ are the same as those used by Kurth et al. [2017b]. That the emissions intensify close to $f_{c e}$ is consistent with generation by the cyclotron maser instability (CMI) [Wu and Lee, 1979; Treumann, 2006]. The locations of the possible sources are identified in Figures 1 and 2 with the same letters as in Figure 3. While the possible sources identified here are not at exactly the same frequencies as those examined via Ulysses direction-finding studies [Ladreiter et al., 1994], the locations are qualitatively similar to those found by Ulysses.

Figure 4 shows high resolution burst mode Waves data for this source showing emission very close to and even below $f_{c e}$. Louarn et al. [2017] have assessed the electron distribution function observed by the Jovian Auroral Distributions Experiment (JADE) plasma instrument in conjunction with Event $\mathrm{C}$ and have concluded a loss cone found there is sufficient to drive the CMI. The burst mode data also show significant spectral structure in the hectometric radiation near the source, similar to that observed in Auroral Kilometric Radiation at Earth [Gurnett et al., 1979a] and Saturn Kilometric Radiation [Kurth et al., 2005]. Imai et al. [2017b] showed direction finding results for broadband kilometric radiation including a source near the position of Event $\mathrm{A}$ in Figure 3.

Additional early results from Juno observations of Jovian auroral radio emissions include studies of the latitudinal beaming of decametric emissions using Juno and the Nançay Decametric Array [Imai et al., 2017a] as well as through statistical studies from early Juno observations [Imai et al., 2017c]. Louis et al. [2017] showed the ability of the 


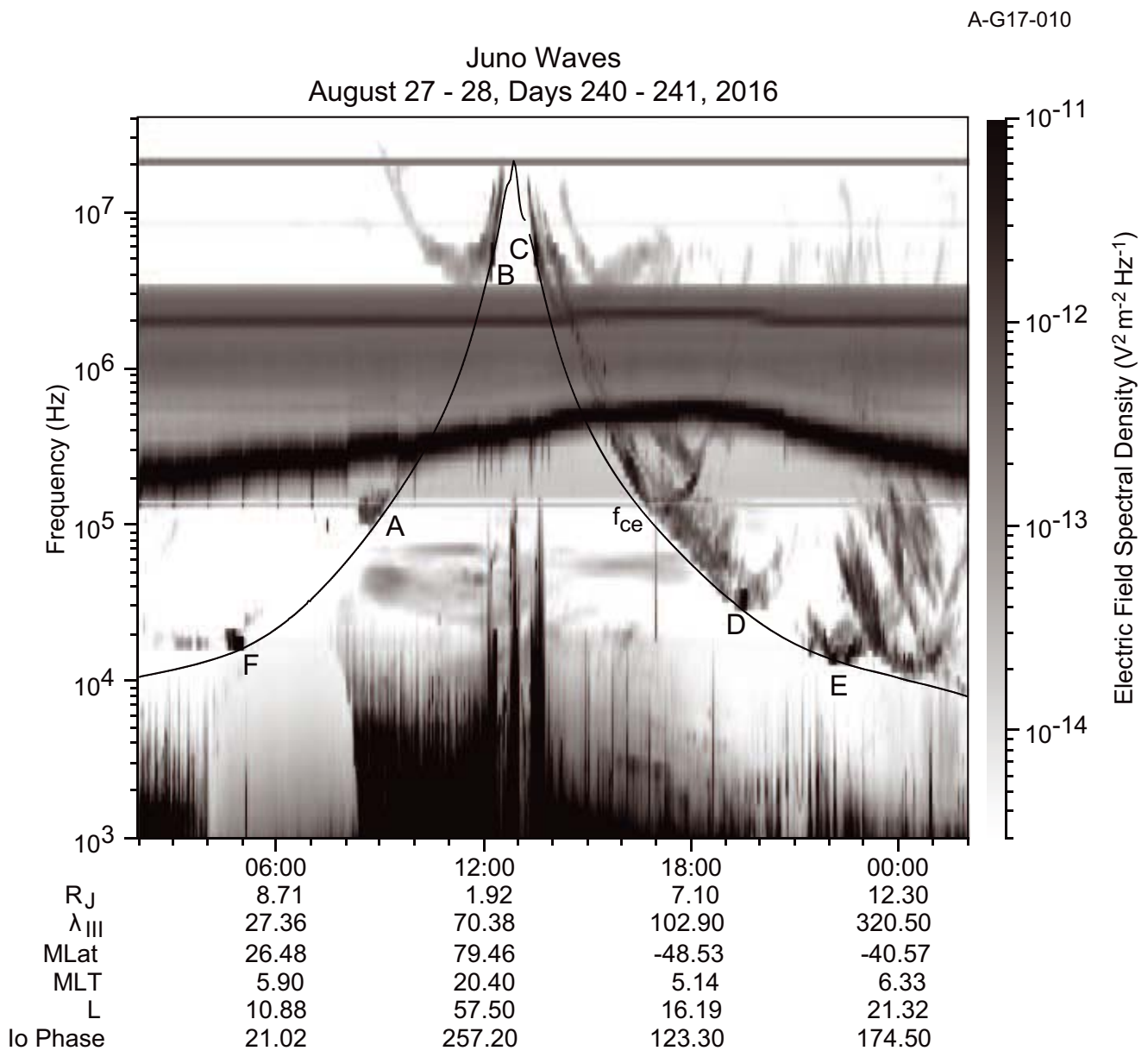

Figure 3: Close or in-situ observations of auroral radio emission sources, similar to Kurth et al. [2017b].

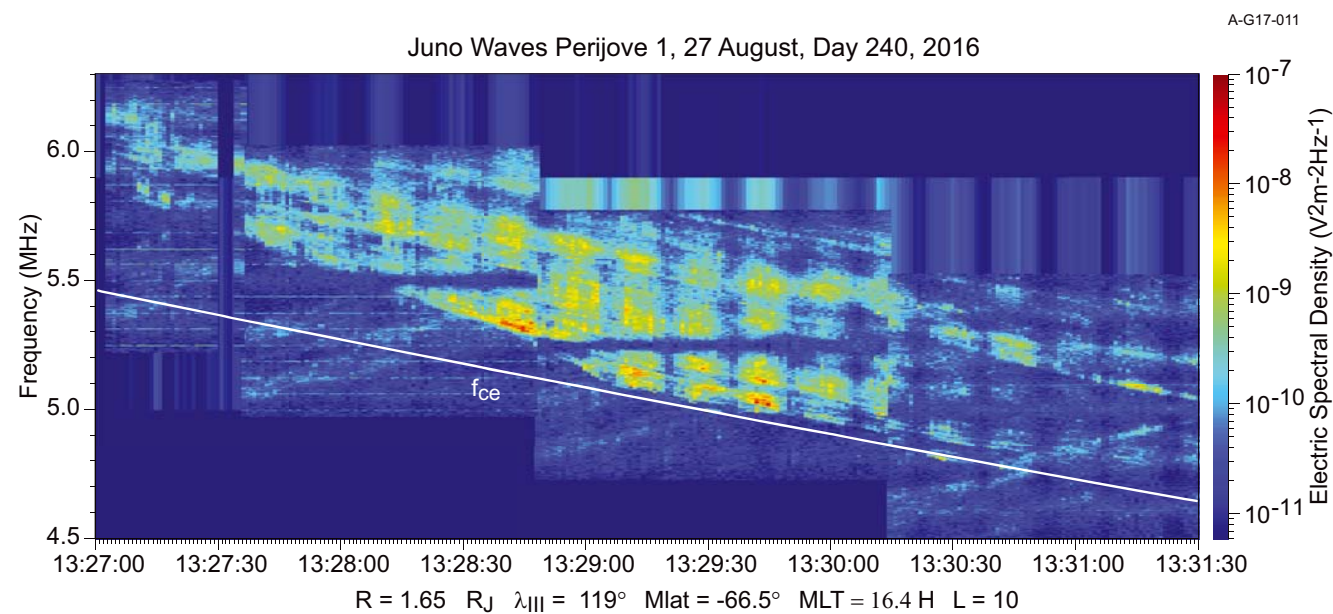

Figure 4: High resolution observations superposed on survey data for Event C.

ExPRES simulation tool to reproduce Io-related decametric arcs observed by Juno. 


\section{Plasma waves observed in Jupiter's polar magnetosphere}

Juno observed strong plasma waves at frequencies below about $20 \mathrm{kHz}$ over both polar caps and associated with Jupiter's main auroral oval. Figure 5 shows electric (top panel) and magnetic (bottom panel) frequency-time spectrograms for the interval roughly centered on Perijove 1. In the northern polar cap, the spectrum is dominated by electromagnetic emissions identified by Tetrick et al. [2017] as whistler-mode hiss. This identification depends on $E / c B$ field ratios near 1 over much of the emission with the exception of larger ratios near the funnel-shaped features near 08:30 and 12:00 UT. These features are reminiscent of auroral hiss funnels in Earth's auroral regions [Gurnett, 1966] where the whistler-mode emissions propagate near the resonance cone and tend to larger values of $E / c B$. They also show that the waves have a polarization consistent with that expected for whistler mode emissions. The identification relies on the assumption that the electron plasma frequency $f_{\text {pe }}$ lies in the range of $20-40 \mathrm{kHz}$ over the northern polar region.

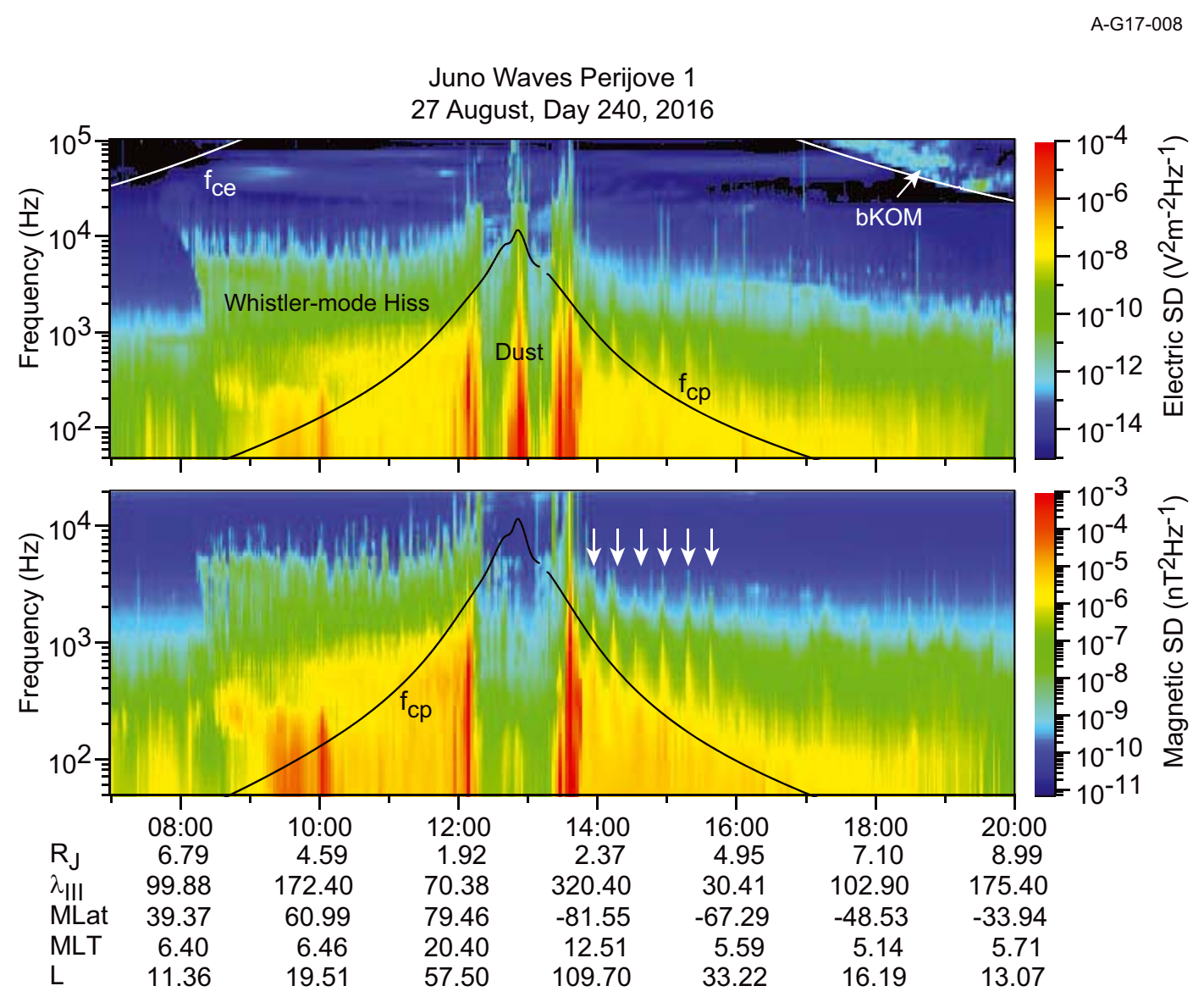

Figure 5: Observations of whistler-mode hiss, dust, and other features in the polar regions on Perijove 1.

Tetrick et al. [2017] showed a good correlation between the whistler-mode hiss over the north polar cap and upgoing beams of energetic electrons observed by the Juno Energetic particle Detector (JEDI) reported by Mauk et al. [2017]. While not always the case, sometimes the hiss shows periodic variations in intensity. A series of evenly spaced (in 
time) arrows are superposed in the bottom panel of Figure 5 to illustrate the existence of periodicities in the hiss in the southern hemisphere. The period of these emissions is about 20 minutes, but other periods have been observed at other times. Twenty minutes is within the range of periods reported for a number of phenomena in Jupiter's magnetosphere including quasi-periodic (QP) radio bursts [MacDowall et al., 1993], X-ray bursts [Gladstone et al., 2002], and pulsations in polar auroras [Bonfond et al., 2011]. Hence, suspicion is aroused that these periodicities are a related phenomena, although the connection of all of these QP phenomena is neither confirmed nor understood at this time. However, the correlation between the hiss intensity and the energetic electron beams shown by Tetrick et al. [2017] albeit at a different time, certainly suggests the electron beams over the polar cap may be involved in some way.

\section{Dust and other observations near Jupiter's equator}

For the segment of Juno's trajectory from northern mid-latitudes through the equator to southern mid-latitudes waveforms from the Waves $50 \mathrm{~Hz}$ to $20 \mathrm{kHz}$ electric channel were recorded in anticipation of impacts by dust grains falling from Jupiter's ring system into the atmosphere. Signatures of such impacts have been used on many missions to study dust in various locations in the solar system [cf. Gurnett et al., 1983; Ye et al., 2016]. The waveform recording for Perijove 1 collects a series of 6144 16-bit samples at a rate of $50 \mathrm{kSps}$ and a duty cycle of $123 \mathrm{~ms}$ every 2 seconds. These observations are shown in Figure 6. The top panel in this figure shows the frequency-time spectrogram resulting from a Fourier transform of the waveforms on the ground. The intense broadband feature, particularly centered at about 12:52:30 UT is primarily due to dust centered near the jovigraphic equator. Interesting, however, is the secondary peak centered around 12:55:30 UT that is well south of the equator at a latitude of about $-8.6^{\circ}$. It should be noted that the magnetic equator as determined from the VIP4 dipole is traversed at 12:51:15 UT. The middle panel of the figure is a highly compressed waveform which serves primarily to highlight these two dust concentrations while the bottom panel shows a 123-ms waveform selected to show sample impact waveforms from about 12:52:04 UT. A very preliminary assessment of such impact signatures gives an impact rate of a few per second. Additional analyses should provide a size distribution for the dust.

Other features are visible in the upper panel of Figure 6. There is a feature most prominent between about 13:03 and 13:08 in the range of about 5 to $8 \mathrm{kHz}$. The bursty nature and varying frequency is highly suggestive of electron plasma oscillations. If so, their frequency provides a measure of the electron density at this location in the magnetosphere of $\sim 0.3$ to $0.8 \mathrm{~cm}^{-3}$. Similar features appear around 12:35 to 12:42 UT, but the case is less clear that these are plasma oscillations. Also, there is a smooth cutoff below the plasma oscillations from about 13:02 and 13:05 UT near $5 \mathrm{kHz}$ and also around $8 \mathrm{kHz}$ at 12:42 UT. To a high degree of accuracy, this cutoff is at the proton cyclotron frequency. This is highlighted with a white trace at $f_{\mathrm{cp}}$, but shifted downward by $500 \mathrm{~Hz}$ so that the cutoff can be seen. Above about $10 \mathrm{kHz}$ before 12:45 and after 13:03 there are wispy features. If we assume the identification of plasma oscillations is correct, these would be above the electron plasma frequency and below the electron cyclotron frequency (which is in the $\mathrm{MHz}$ range 
during this interval). Hence, we conclude that these wispy emissions are either ordinary mode radio emissions or Z-mode emissions.
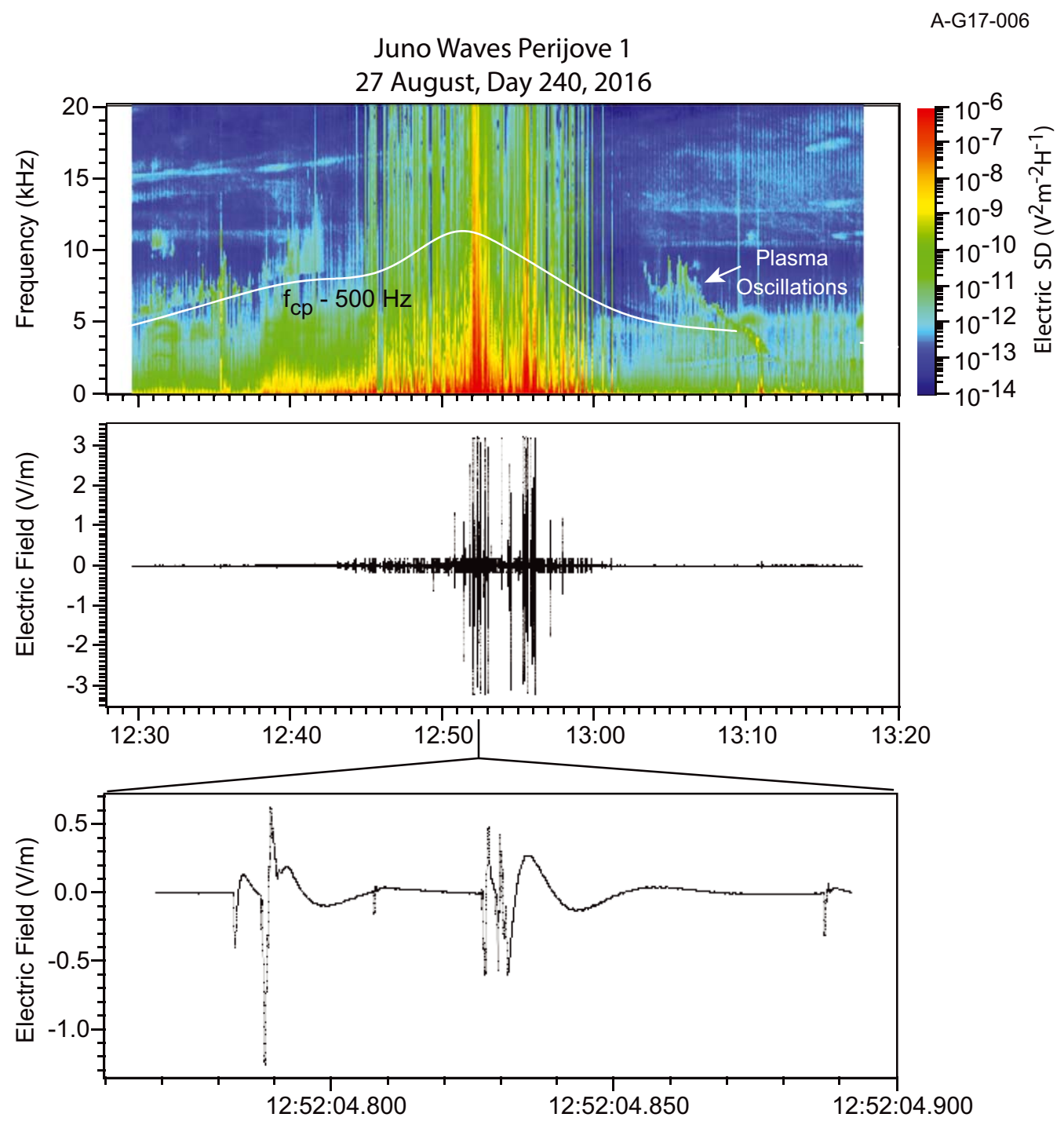

Figure 6: Evidence of dust near the jovigraphic equator.

\section{$5 \quad$ Lightning whistlers}

The existence of lightning on Jupiter was discovered by the detection of lightning whistlers by Voyager 1 [Gurnett et al., 1979b] and by clouds illuminated from below by lightning [Cook et al., 1979]. Voyager's sensitivity to lightning was limited by the amount of other naturally-occurring emissions in the Io torus [Kurth et al., 1985]. Lightning typically occurs at Jupiter near $50^{\circ}$ jovigraphic latitude [Little et al., 1999], hence, Juno has an excellent possibility to cross magnetic field lines threading thunderstorms during its rapid latitudinal passage near perijove. Figure 7 a shows an example of whistlers observed 
during Juno's first perijove pass. The remarkable aspect of these whistlers is the very small dispersion; the typical duration is of order $10 \mathrm{~ms}$, compared with a few seconds for those observed by Voyager. This is because of the very small column density and strong magnetic field along the whistler path from the lightning stroke to Juno. Figure 7b shows an example of a proton whistler [Gurnett et al., 1965]. These immediately follow an electron whistler in which the dispersion results in an initial rapid rise in frequency and asymptotically approaching the proton cyclotron frequency. This is the first detection of a proton whistler other than at Earth.
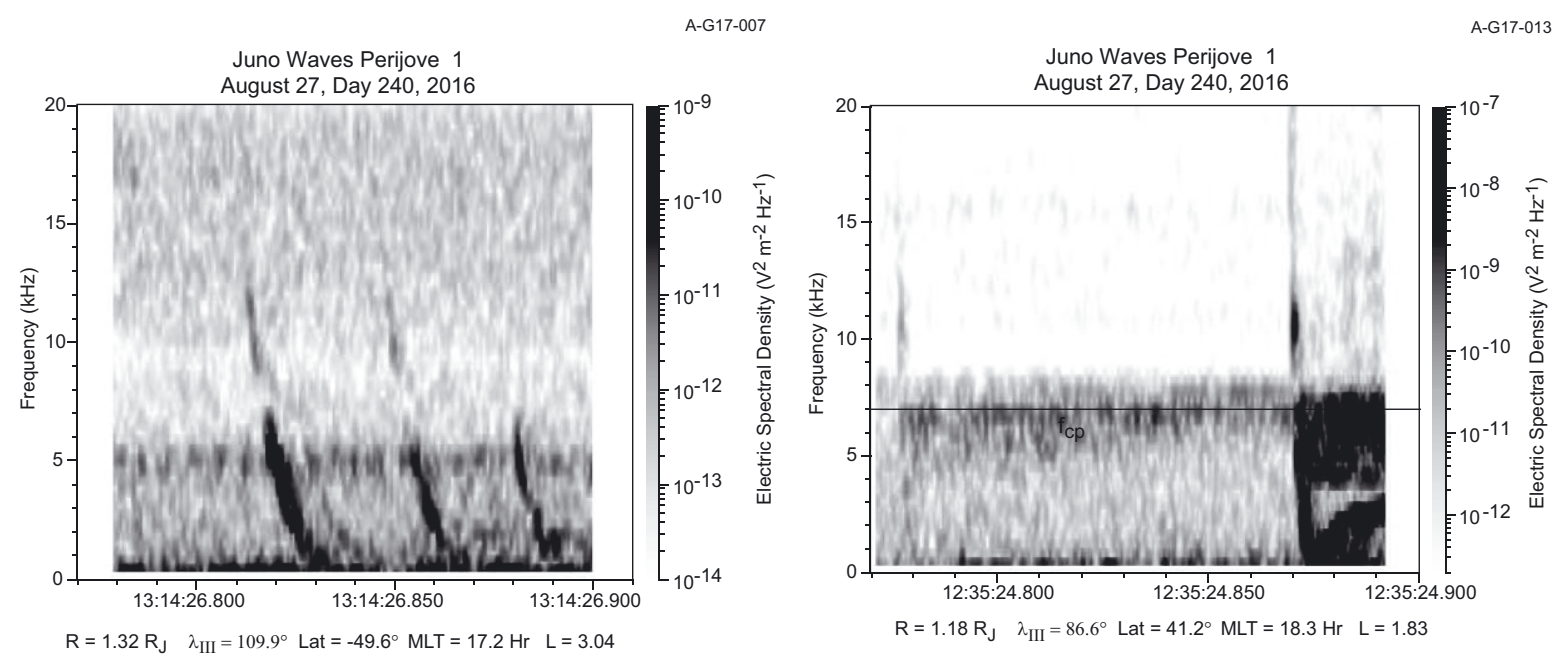

Figure 7: (a) Examples of low-dispersion whistlers near Perijove 1. (b) Example of proton whistler. The line labeled $f_{c p}$ is the proton cyclotron frequency.

\section{Conclusions}

Juno has already provided significant insight into Jupiter's auroral radio emissions and plasma waves. In just the first perijove, it has crossed through or very close to five or six radio emission sources, provided a view of the entire auroral radio spectrum from high latitudes, and provided the first examples of the electron distribution functions responsible for driving the cyclotron maser instability. Juno has found evidence for whistler mode hiss associated with upgoing electron beams, observed whistlers directly over thunderstorms, and sampled the dust population presumably dropping out of the ring system into the atmosphere.

This paper presents just an overview of early results from the Juno Waves investigation of Jupiter's inner magnetosphere. The Juno mission plan calls for more than 30 orbits and a number of the topics introduced herein will benefit by multiple perijoves. Additionally, the line of apsides of Juno's orbit rotates about one degree each orbit, such that apojove moves south and perijove moves north. One important aspect of this precession is that the trajectory will cross the aurora oval and polar cap at progressively lower altitudes in the north and progressively higher altitudes in the south, providing a comprehensive examination of auroral field lines over a wide range of altitudes. Juno's mission plan 
called for a period reduction maneuver to reduce the orbit period to 14 days. An issue with the propulsion system led to the decision to not do this maneuver, since the periapsis science is basically unaffected by the longer orbital period. An advantage of the 53-day orbit duration is that there will be a significant progression of local time by the orbit plane, eventually passing through local midnight and into the post-dusk sector. The net result is an orbital tour that will provide a great survey of not only Jupiter's polar magnetosphere, but also portions of the magnetosphere that have not been sampled to any significant degree.

Acknowledgments. The research at the University of Iowa is supported by NASA through Contract 699041X with the Southwest Research Institute. The Editors thank two anonymous reviewers for their help in evaluating this paper.

\section{References}

Bonfond, B., M. F. Vogt, J.-C. Gérard, D. Grodent, A. Radioti, and V. Coumans, Quasiperiodic polar flares at Jupiter: A signature of pulsed dayside reconnections? Geophys. Res. Lett., 380, L02104, 2011.

Connerney, J. E. P., M. H. Acuña, and N. F. Ness, Modeling the Jovian current sheet and inner magnetosphere, J. Geophys. Res., 86, 8370-8374, 1981.

Connerney, J.E.P., M. H. Acuña, N.F. Ness, and T. Satoh, New models of Jupiter's magnetic field constrained by the Io flux tube footprint, J. Geophys. Res., 103, A6, 11929-11939, 1998.

Connerney, J. E. P., A. Adriani, F. Allegrini, F. Bagenal, S. J. Bolton, B. Bonfond, S. W. H. Cowley, J.-C. Gérard, G. R. Gladstone, D. Grodent, G. Hospodarsky, J. L. Jorgensen, W. S. Kurth, S. M. Levin, B. Mauk, D. J. McComas, A. Mura, C. Paranicas, E. J. Smith, R. M. Thorne, P. Valek, and J. Waite, Jupiter's magnetosphere and aurorae observed by the Juno spacecraft during its first polar orbits, Science, 356, 826-832, 2017.

Cook, A.F., T. C. Duxbury, and G.E. Hunt, First results on Jovian lightning, Nature, 280, p.794, 1979.

Gladstone, G. R., J. H. Waite, D. Grodent, W.S. Lewis, F. J. Crary, R. F. Elsner, M. C. Weisskopf, T. Majeed, J.-M. Jahn, A. Bhardwaj, J. T. Clarke, D. T. Young, M. K. Dougherty, S. A. Espinosa, and T.E. Cravens, A pulsating auroral X-ray hot spot on Jupiter, Nature, 415, 1000-1003, 2002.

Gurnett, D. A., A satellite study of VLF hiss, J. Geophys. Res., 71, 5599-5615, 1966.

Gurnett, D. A., S. D. Shawhan, N. M. Brice, and R. L. Smith, Ion cyclotron whistlers, J. Geophys. Res., 70, 1665-1688, 1965.

Gurnett, D. A., R. R. Anderson, F. L. Scarf, R. W. Fredricks, and E. J. Smith, Initial results from the ISEE-1 and -2 plasma wave investigation, Space Sci. Rev., 23, 103-122, 1979a. 
Gurnett, D. A., R. R. Shaw, R. Anderson, and W. S. Kurth, Whistlers observed by Voyager 1: Detection of lightning on Jupiter, Geophys. Res. Lett., 6, 511-514, 1979b.

Gurnett, D. A., E. Grün, D. Gallagher, W.S. Kurth, and F. L. Scarf, Micron-sized particles detected near Saturn by the Voyager Plasma Wave Instrument, Icarus, 53, 236-254, 1983.

Gurnett, D. A., W.S. Kurth, A. Roux, S. J. Bolton, and C. F. Kennel, Galileo plasma wave observations in the Io plasma torus and near Io, Science, 274, 391-392, 1996.

Gurnett, D. A., et al. (16 co-authors), Control of Jupiter's radio emission and aurorae by the solar wind, Nature, 415, 985-987, 2002.

Hess, S., E. Echer, P. Zarka, L. Lamy, and P. Delamere, Multi-instrument study of the Jovian radio emissions triggered by solar wind shocks and inferred magnetospheric subcorotation rates, Planet. Space Sci., 99, 136-148, 2014.

Hospodarsky, G. B., W. S. Kurth, S. J. Bolton, F. Allegrini, G. B. Clark, J. E. P. Connerney, R. W. Ebert, D. K. Haggerty, S. Levin, D. J. McComas, C. Paranicas, A. M. Rymer, and P. W. Valek, Jovian bow shock and magnetopause encounters by the Juno spacecraft, Geophys. Res. Lett., 44, 4506-4512, 2017.

Imai, M., W.S. Kurth, G. B. Hospodarsky, S. J. Bolton, J. E. P. Connerney, S. M. Levin, A. Lecacheux, L. Lamy, and P. Zarka, Latitudinal beaming of Jovian decametric radio emissions as viewed from Juno and the Nançay Decameter Array, Geophys. Res. Lett., 44, 4455-4462, 2017a.

Imai, M., W. S. Kurth, G. B. Hospodarsky, S. J. Bolton, J. E. P. Connerney, and S. M. Levin, Direction-finding measurements of Jovian low-frequency radio components by Juno near Perijove 1, Geophys. Res. Lett., 44, 6508-6516, $2017 \mathrm{~b}$.

Imai, M., W. S. Kurth, G. B. Hospodarsky, S. J. Bolton, J. E. P. Connerney, and S. M. Levin, Statistical study of latitudinal beaming of Jupiter's decametric radio emissions using Juno, Geophys. Res. Lett., 44, 4584-4590, 2017c.

Kurth, W.S., B.D. Strayer, D. A. Gurnett, and F. L. Scarf, A summary of whistlers observed by Voyager 1 at Jupiter, Icarus, 61, 497-507, 1985.

Kurth, W.S., G. B. Hospodarsky, D. A. Gurnett, B. Cecconi, P. Louarn, A. Lecacheux, P. Zarka, H. O. Rucker, M. Boudjada, and M. L. Kaiser, High spectral and temporal resolution observations of Saturn kilometric radiation, Geophys. Res. Lett., 32, L20S07, 2005.

Kurth, W. S., G. B. Hospodarsky, D. L. Kirchner, B. T. Mokrzycki, T. F. Averkamp, W. T. Robison, C. W. Piker, M. Sampl, and P. Zarka, The Juno Waves investigation, Space Sci. Rev., 213, 347-392, doi:10.1007/s11214-017-0396-y, 2017a.

Kurth, W.S., M. Imai, G. B. Hospodarsky, D. A. Gurnett, P. Lourarn, P. Valek, F. Allegrini, J. E. P. Connerney, B. H. Mauk, S. J. Bolton, S. M. Levin, A. Adriani, F. Bagenal, G. R. Gladstone, D. J McComas, and P. Zarka, A new view of Jupiter's auroral radio spectrum, Geophys. Res. Lett., 44, 7114-7121, doi: 10.1002/2017GL072889, $2017 \mathrm{~b}$. 
Ladreiter, H. P., P. Zarka, and A. Lecacheux, Direction finding study of Jovian hectometric and broadband kilometric radio emissions: Evidence for their auroral origin, Planet. Space Sci., 42, 919-931, 1994.

Little, B., C. D. Anger, A. P. Ingersoll, A. R. Vasavada, D. A. Senske, H. H. Breneman, W. J. Borucki, and the Galileo SSI Team, Galileo images of lightning on Jupiter, Icarus, 142, 306-323, 1999.

Louarn, P., F. Allegrini, D. J. McComas, P. W. Valek, W. S. Kurth, N. André, F. Bagenal, S. Bolton, J. Connerney, R. W. Ebert, M. Imai, S. Levin, J. R. Szalay, S. Weidner, R. J. Wilson, and J. L. Zink, Generation of the Jovian hectometric radiation: First lessons from Juno, Geophys. Res. Lett., 44, 4439-4446, 2017.

Louis, C. K., L. Lamy, P. Zarka, B. Cecconi, M. Imai, W.S. Kurth, G. Hospodarsky, S. L. G. Hess, X. Bonnin, S. Bolton, J. E. P. Connerney, and S. M. Levin, Io--Jupiter decametric arcs observed by Juno/Waves compared to ExPRES simulations, Geophys. Res. Lett., 44, 9225-9232, doi:10.1002/2017GL073036, 2017.

MacDowall, R. J., M. L. Kaiser, M. D. Desch, W. M. Farrell, R. A. Hess, and R. G. Stone, Quasi-periodic Jovian radio bursts: Observations from the Ulysses radio and plasma wave experiment, Planet Space Sci., 41, 1059-1072, 1993.

Mauk, B.H., et al. (16 co-authors), Juno observations of energetic charged particles over Jupiter's polar regions: Analysis of monodirectional and bidirectional electron beams, Geophys. Res. Lett., 44, 4410-4418, 2017.

Scarf, F. L., D. A. Gurnett, and W. S. Kurth, Jupiter plasma wave observations: An initial Voyager 1 overview, Science, 204, 991-995, 1979.

Stone, R. G., B. M. Pedersen, C. C. Harvey, P. Canu, N. Cornilleau-Wehrlin, M. D. Desch, C. de Villedary, J. Fainberg, W. M. Farrell, and K. Goetz, Ulysses radio and plasma wave observations in the Jupiter environment, Science, 257, 1524-1531, 1992.

Tetrick, S.S. D. A. Gurnett, W.S. Kurth, M. Imai, G. B. Hospodarsky, S. J. Bolton, J.E. P. Connerney, S. M. Levin, and B. H. Mauk, Plasma waves in Jupiter's high latitude regions: Observations from the Juno spacecraft, Geophys. Res. Lett., 44, 4447-4454, 2017.

Treumann, R.A., The electron-cyclotron maser for astrophysical application, Astron. Astrophys. Rev., 13, 229-315, 2006.

Warwick, J. W., J. B. Pearce, A. C. Riddle, J. K. Alexander, M. D. Desch, M. L. Kaiser, J. R. Thieman, T. D. Carr, S. Gulkis, A. Boischot, C. C. Harvey, and B. M. Pedersen, Voyager-1 Planetary Radio Astronomy observations near Jupiter, Science, 204, 995998, 1979.

Wu, C.S., and L. C. Lee, A theory of terrestrial kilometric radiation, Astrophys. J., 230, 621-626, 1979.

Ye, S.-Y, W.S. Kurth, G. B. Hospodarsky, T.F. Averkamp, and D. A. Gurnett, Dust detection in space using the monopole and dipole electric field antennas, J. Geophys. Res., 121, 11964-11972, 2016. 